\title{
MARCO CONTEXTUAL DEMOGRÁFICO DE LA EDUCACIÓN SUPERIOR EN LA RED EDUCATIVA UNAH DE LA REGION SUR
}

\author{
Manuel Antonio Flores Fonseca, Universidad Nacional Autónoma de Honduras, \\ Instituto de Investigaciones Económicas y Sociales (IIES UNAH) \\ Ciudad Universitaria edificio C2 primer piso. Tel./Fax: 504-22391849 \\ Correo electrónico: mflores@iies-unah.org
}

\section{RESUMEN}

Este artículo surge dentro del proyecto de investigación sobre "Oferta y Demanda de la Educación Superior en Honduras" que realiza la Universidad Nacional Autónoma de Honduras (UNAH), y que pretende estudiar la educación terciaria para contribuir a un mejor balance entre la oferta y la demanda por medio de un sistema integrado de valor y suministro de la educación superior. Específicamente se pretende estudiar la cadena de valor que tiene el beneficiario/demandante/usuario de educación superior como la fuente del valor, que significa un flujo de valor en forma de demanda, desde el demandante (final e intermedio), hasta el ofertante (ES), que incluye a su proveedor de insumos. Dicho flujo o cadena de demanda se manifiesta por el número de peticiones de graduados, como también por los resultados de investigaciones y dinero paralelo al flujo de valor, fluyendo hacia atrás (en dirección opuesta al flujo de la oferta). La cadena de suministro, es un flujo hacia adelante, en forma de oferta, desde el ingreso de estudiantes y propuestas de investigación, como los insumos provistos a la fuente de oferta, hasta el demandante/beneficiario intermedio y final.

Dicha investigación por su complejidad se está desarrollando en varias etapas y dentro de las primeras en la contextualización del entorno socioeconómico, uno de ellas es la contextualización demográfica de la educación superior a nivel nacional, a su vez a nivel regional se operacionaliza en nueve regiones a través de las redes educativas regionales de la UNAH. El presente trabajo constituye la contextualización demográfica de la Red Educativa UNAH de la Región Sur, que abarca los departamentos de Choluteca y Valle y cuya sede central es el Centro Universitario del Litoral Pacífico (CURLP) con sede en la Ciudad de Choluteca. Comprende el estudio del tamaño y ritmo de crecimiento de la población, estructura por edad y sexo, comportamiento de las principales variables demográficas como son fecundidad, mortalidad y migración (interna e internacional), distribución 
espacial y varios aspectos de la población en edad de estudiar la educación superior en la región sur.

La población de la región sur continua creciendo aceleradamente, teniendo una estructura joven, los niveles de la fecundidad y mortalidad son altos, comparados con otras regiones del país y la migración interna e internacional continuará haciendo a la región expulsora de población. En cuanto a la población en edad de estudiar en la educación superior ésta continúa creciendo, principalmente en las zonas rurales, sin embargo, los mayores volúmenes a nivel municipal se encuentran en los más poblados, que también son los más urbanizados.

Palabras Clave: Educación Superior, Aspectos demográficos. 


\section{DEMOGRAPHIC CONTEXT FRAMEWORK OF HIGHER EDUCATION IN EDUCATIONAL NETWORK OF SOUTH REGION UNAH}

Manuel Antonio Flores Fonseca, Universidad Nacional Autónoma de Honduras, Instituto de Investigaciones Económicas y Sociales (IIES UNAH) Ciudad Universitaria edificio C2 primer piso. Tel./Fax: 504-22391849 Correo electrónico: mflores@iies-unah.org

\section{ABSTRACT}

This article arises within the research project about "Supply and Demand of Higher Education in Honduras" made by the National Autonomous University of Honduras (UNAH), and attemps to study tertiary education to contribute to a better balance between supply and demand by means of an integrated system of value and supply of higher education. Specifically, it attempts to study the value chain consisting of benefactor-claimer-user of higher education as the source of value, which means a value flow in the form of demand, coming from the one who demands (final or intermediate), to the one who offers, or supplies. This flow or chain of demand manifests itself by the number of graduate petitions, and by research investigation results and money parallel to the flow of value, flowing backwards (in contrast to the flow of supply). This supply chain, is a forwards flow, in the form of an offer, from the inputs of students and research proposals, such as the income to the supply source, to the one who demands or final/intermediate benefactor.

Due to its complexity, this research is being developed in several stages and within the first, in the context of the socioeconomic environment, lies the demographic context of higher education in a national scale, and on a regional scale operation in nine regions through regional education networks of the UNAH. This research work constitutes the demographic contetx of UNAH's South Region Educational Network, which covers the departments of Choluteca and Valle and whose central headquarters is the Pacific Coast University Center (CURLP), in the city of Choluteca. It covers the study of size and growth rate of population, age and gender structure, behavior of main demographic variables such as fertility, mortality, and migration (internal and international), spatial distribution and several aspects of population already in age able to study higher education in the south region. 
The population in the south region continues to grow at an accelerated rate, having a young structure, levels of fertility and mortality are high, in comparison to other regions of the country and internal and international migration keeps making this a rather ejector region. On population in age able to receive higher education, this continues to grow, specially in rural areas, however, the largest concentration of these areas are in the most populated ones, which are also the ones more urbanized.

Key words: Higher Education, Demographic Aspects 


\section{INTRODUCCIÓN.}

El presente informe es una recopilación de las principales variables e indicadores de diversos aspectos que conforman un marco contextual demográfico de la educación superior para la región sur de Honduras, específicamente la red educativa sur de la Universidad Nacional Autónoma de Honduras (UNAH). Esta red incluye los departamentos de Choluteca y Valle, coordinados por el Centro Universitario Regional del Litoral Pacífico (CURLP) con sede en la Ciudad de Choluteca (UNAH, 2008).

Los aspectos a considerar son relativos a la parte demográfica e incluyen el tamaño, ritmo de crecimiento, estructura por edad y sexo de la población, comportamiento de las principales variables demográficas como son fecundidad, mortalidad y migración, incluyendo la interna e internacional; distribución espacial de la población y aspectos relativos a la población en edad de educación terciaria $^{1}$ en la región.

Todos estos elementos tienen por objetivo conocer las principales tendencias demográficas en los aspectos vinculantes con la educación terciaria como elemento de base de contexto de la región en la investigación de Oferta y Demanda de la Educación Superior en Honduras, Sistema Integrado de Valor y Suministro que coordina el Instituto de Investigaciones Económicas y Sociales (IIES) de la Universidad Nacional Autónoma de Honduras (UNAH, 2010).

\section{OBJETIVOS DEL TRABAJO.}

El objetivo es contribuir en la investigación de la oferta y demanda de la educación superior de Honduras a través de insumos de contextualización del tema, específicamente del escenario demográfico, pasado, presente y futuro de la región educativa sur de la UNAH.

\section{METODOLOGÍA.}

La metodología utilizada es descriptiva y parte de construcciones de los indicadores demográficos

1 La educación terciaria, es usada como sinónimo de la educación superior, enseñanza superior, estudios superiores y se refiere al proceso, los centros y las instituciones educacionales que están después de la educación secundaria o media. 
principales del pasado y presente, así como de algunas estimaciones a futuro; todas ellas dependiendo de la disponibilidad de información en las fuentes publicadas, por lo que en su mayoría fue necesario la generación de información específica de la región en bases de datos demográficas originales.

Se utilizó como fuentes demográficas, los censos de población, encuestas demográficas y proyecciones de población, elaboradas por la anterior Dirección de Estadísticas y Censos de Honduras (DGECH) y ahora Instituto Nacional de Estadística de Honduras (INE).

\section{RESULTADOS}

\subsection{Tamaño y ritmo de crecimiento.}

La región sur (Choluteca y Valle) ha tenido un crecimiento demográfico lento desde el siglo diecinueve hasta los años cincuenta del siglo pasado. A partir de ese año se experimenta un crecimiento acelerado de la población, mismo que en esos años no superaba las 180 mil personas hasta alcanzar en el año 2011 una población de 641,029 personas, es decir aumentó en 3.7 veces la población en sesenta años (Gráfico No.1).

Este aumento es general en el país y la región no escapa a ello. Es producto de la baja pronunciada de la mortalidad a raíz de las mejoras y acceso a los servicios de salud, y la introducción de medicamentos de bajo costo. La natalidad ha sido alta, lo cual ha generado una alta tasa de crecimiento de la población (Flores, 2004).

La tasa de crecimiento demográfico estimada para la región entre los períodos censales muestra cambios de comportamiento entre 1.6 y 2.6 por ciento. El crecimiento del periodo 2001-2011 se ubica en 2.4 por ciento, considerado todavía alto demográficamente.

Para el 2011, la región sur tenía una densidad demográfica de 109.3 habitantes por kilómetro cuadrado. 


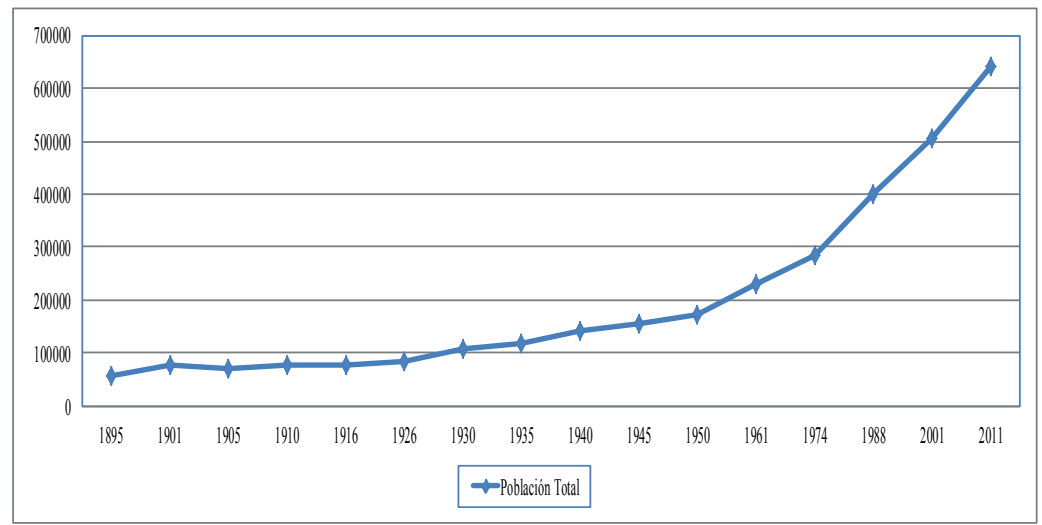

Gráfico No. 1

Red Sur UNAH: Población total según censo y proyección

(Fuente: Elaboración propia en base a datos censales de DGECH e INE, Honduras.)

En las últimas décadas el índice de masculinidad en la región sur es de alrededor de 98 por ciento, indicando que hay más mujeres que hombres, lo cual es una tendencia nacional, aun cuando la región es eminentemente rural, con características semiurbanas, donde generalmente habría una mayor proporción de hombres.

\subsection{Estructura por edad y sexo.}

Según la pirámide de población, los datos por edad y sexo muestran las transformaciones que han sucedido en la estructura demográfica y la tendencia al paso de una pirámide expansiva a una constrictiva que culminará en el mediano y largo plazo (Gráfico No.2).

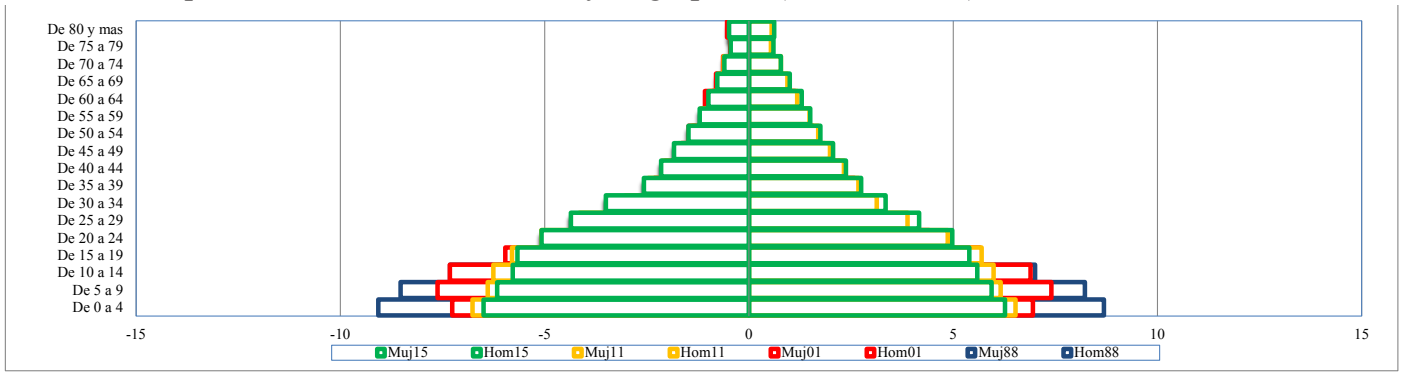

Gráfico No. 2

Red Sur UNAH: Pirámides de población, 1988-2015

(Fuente: Elaboración propia en base a datos censales de DGECH e INE, Honduras.) 
La población de la región es joven, donde el 38\% no supera los quince años, sin embargo, el mayor porcentaje de población se ubica en la edad de trabajar (activa) y en menor medida se encuentra la población de la tercera edad (Tabla No.1). En estos tres grandes grupos de edad se puede observar las transformaciones de la estructura demográfica hacia poblaciones activas y poblaciones adultas mayores, producto principalmente de la tendencia de la baja en la fecundidad y también de la mortalidad.

\section{Tabla No.1}

Red Sur UNAH: Población por Grupos de Edad, 1988, 2001, 2011

\begin{tabular}{|l|r|r|r|}
\hline \multicolumn{1}{|c|}{ Grupos de Edad } & \multicolumn{1}{|c|}{1988} & \multicolumn{1}{c|}{2001} & \multicolumn{1}{c|}{2011} \\
\hline $0-14$ años & 48.83 & 43.29 & 38.10 \\
\hline $15-64$ años & 47.13 & 51.45 & 56.21 \\
\hline 65 años y más & 4.04 & 4.87 & 5.69 \\
\hline Total & 100.00 & 100.00 & 100.00 \\
\hline
\end{tabular}

(Fuente: Cálculos propios en base a datos de Censos de Población y Proyecciones, DGECH e INE.)

También las transformaciones de la estructura por edad se manifiestan en las relaciones de dependencia, tanto en la total $\left(\mathrm{RDT}^{2}\right)$, de la niñez $\left(\mathrm{RDN}^{3}\right)$ y de la vejez $\left(\mathrm{RDV}^{4}\right)$. En las primeras dos se manifiestan los cambios hacia la reducción de este indicador, mientras que en la tercera hay una tendencia hacia el aumento, pero a niveles muy menores.

En la RDT los niveles pasan de 112.2 en 1988 hasta 77.9 en el 2011, lo que indica que hay 78 personas dependientes por cada cien personas en edades productivas. Esto es una situación favorable en el tiempo en cuanto a la reducción de dependientes, sin embargo, el número de personas en edad productiva ha crecido en el tiempo y necesita emplearse para generar beneficios personales, familiares y sociales. Los valores de RDN actualmente son de 68 niños dependientes y 10.1 dependientes adultos mayores por cien en edades productivas (Tabla No.2).

2 Relación de Dependencia Total RDT es la población menor de 15 años más la población de 65 años y más entre la población de 15 a 64 años.

3 Relación de Dependencia de la Niñez RDN es la población menor de 15 años a 64 años.

4 Relación de Dependencia de la vejez RDV es la población de 65 años y más entre la población de 15 a 64 años. 
Tabla No.2

Red Sur UNAH: Relaciones de Dependencia, 1988,2001, 2011.

\begin{tabular}{|l|r|r|r|}
\hline \multicolumn{1}{|c|}{ Relaciones de Dependencia } & 1988 & 2001 & 2011 \\
\hline Relación de Dependencia Total (RDT) & 112.20 & 93.60 & 77.90 \\
\hline Relación de Dependencia de la Niñez (RDN) & 103.63 & 84.13 & 67.77 \\
\hline Relación de Dependencia de la Vejez (RDV) & 8.57 & 9.47 & 10.13 \\
\hline
\end{tabular}

(Fuente: Cálculos propios en base a datos de Censos de Población y Proyecciones, DGECH e INE.)

\subsection{Dinámica demográfica.}

\subsubsection{Fecundidad y mortalidad.}

En la dinámica demográfica de la región sur se observan niveles similares a los de Honduras, con respecto a las tres variables demográficas que son mortalidad, fecundidad y migración. Los indicadores de emigración, sin duda están por encima de la media nacional, provocadas por las tasas de migración interna e internacional.

Entre el 2001 y el 2011 la mayoría de indicadores en esta región han disminuido, a excepción de la esperanza de vida al nacer, la cual ha aumentado de $70.8 \%$ a $73.8 \%$ (Tabla No.3).

El mejoramiento de los indicadores de mortalidad y fecundidad se debe a las mejoras en las condiciones de vida, especialmente de salud, producto de los esfuerzos gubernamentales por incrementar el acceso a los sistemas sanitarios, campañas de vacunación, acceso a agua potable y sistemas de eliminación de excretas, entre otros. 
Tabla No.3

Red Sur UNAH: Principales Indicadores Demográficos, 2001-2011

\section{|}

\begin{tabular}{|l|c|c|c|c|c|c|}
\hline 2001 & TGF & TBN & TBM & INM & Esperanza de Vida & TMI \\
\hline Coluteca & 4.3 & 31.9 & 5.7 & -7.36 & 70.4 & 32.9 \\
\hline Valle & 4.1 & 30.2 & 5.6 & -10.46 & 71.4 & 31.2 \\
\hline $\begin{array}{l}\text { Total } \\
\text { Región }\end{array}$ & 4.2 & 31.05 & 5.65 & -8.91 & 70.9 & 32.1 \\
\hline Honduras & 4.09 & 32.8 & 5.2 & -2.5 & 70.8 & 34.8 \\
\hline 2011 & & & & & & 73.5 \\
\hline Choluteca & 3.5 & 27.8 & 5 & -5.62 & 74.2 & 23.3 \\
\hline Valle & 3.4 & 28.1 & 5.2 & -9.5 & 73.9 & 22.2 \\
\hline $\begin{array}{l}\text { Total } \\
\text { Región }\end{array}$ & 3.5 & 28.0 & 5.1 & -7.56 & & 24.3 \\
\hline Honduras & 3.29 & 27.3 & 4.5 & -2.14 & 73.8 & \\
\hline
\end{tabular}

(Fuente: Proyecciones de Población, INE.)

\subsubsection{Migración interna.}

En relación a este aspecto, la región sur que incluye los departamentos de Choluteca y Valle en las tres últimas décadas ha mostrado ser una región con saldos netos de migración negativos a nivel de División Administrativa Mayor (DAM) , que en Honduras es el departamento, es decir es una región expulsora de población y a través del tiempo este patrón se ha incrementado (Flores, 2005). De la región en la migración absoluta hasta el año 2001 habían salido 152,680 personas y apenas habían ingresado a la región solo 26,469, por lo que el resultado es un saldo migratorio negativo interdepartamental de 126,211 personas, mostrando así a la región como la que pierde población hacia las otras regiones del país.

En la migración interna reciente, en el período 1996-2001, la región también tuvo un saldo migratorio negativo que asciende a 14,068 personas.

A nivel de División Administrativa Menor (DAME), que en el caso de Honduras es el municipio, encontramos que a ese nivel de desagregación los municipios de la región tanto en la migración absoluta como en la reciente tienen saldos migratorios negativos, a excepción de Marcovia en la 
migración absoluta hasta el 2001 y Santa Ana Yusguare en la migración reciente para el período 1996-2001 (Gráficos No. 3 y No.4).

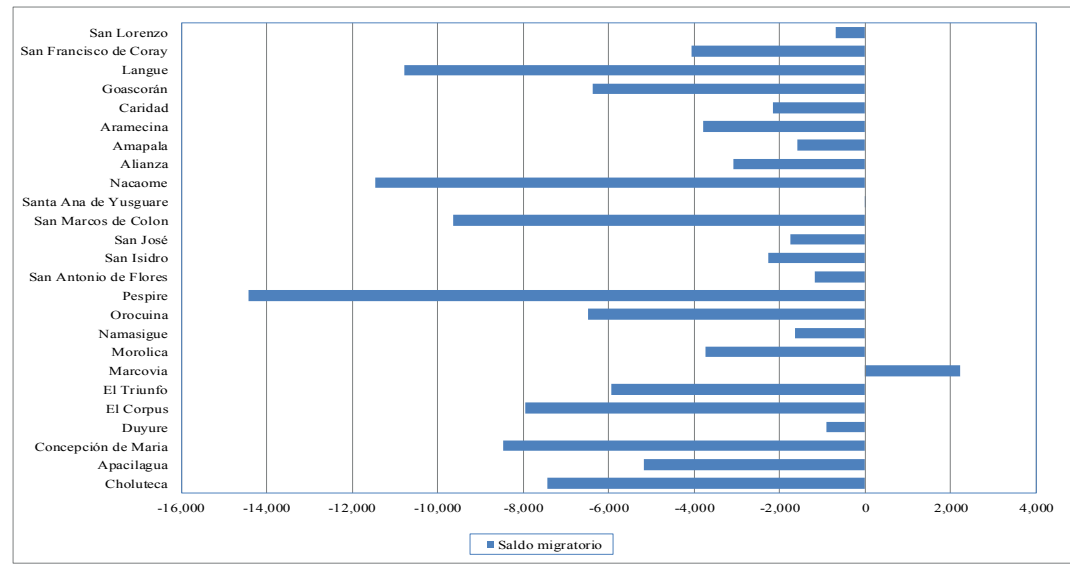

Gráfico No.3

Red Sur UNAH: Saldos migratorios de toda la vida, 2001

(Fuente: Elaboración propia en base de datos censales de CNPV 2001, INE, Honduras)

En la región hay municipios con saldos migratorios negativos de relevancia en cuanto a la migración absoluta hasta el 2001, tal es el caso de Pespire, Nacaome, Langue, San Marcos de Colón, Concepción de María, El Corpus, Choluteca y Orocuina.

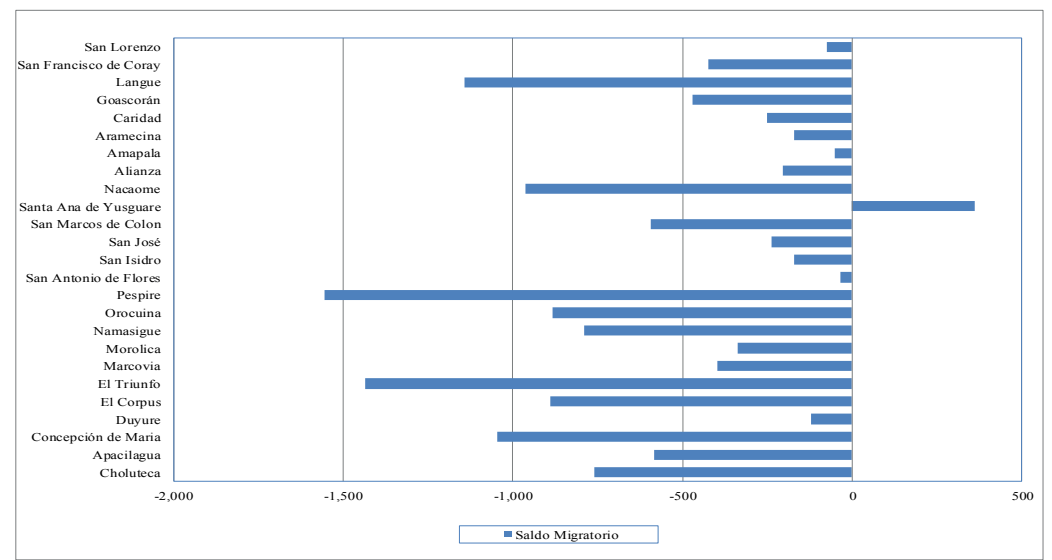

Gráfico No. 4

Red Sur UNAH: Saldo migratorio reciente, 1996-2001

(Fuente: Elaboración propia en base a datos censales del CNPV 2001, INE, Honduras) 
En la migración reciente, aunque en volúmenes menores sobresalen los saldos migratorios negativos de Pespire, El Triunfo, Langue, Concepción de María, Nacaome, Orocuina, Choluteca, que coinciden con la característica de pérdida de población por emigración interna de toda la vida.

Los principales flujos migratorios internos de toda la vida a nivel de DAME desde la región (con cifras mayores de 2,000 personas) hasta el año 2001, proceden principalmente de Choluteca, Pespire, Nacaome, Orocuina, El Corpus, Langue, Texiguat, Reitoca, Concepción de María y de San Lorenzo a Tegucigalpa, de Choluteca a San Pedro Sula, El Corpus a Choluteca. También hay un flujo de Tegucigalpa a Choluteca. En la migración interna reciente (con cifras mayores a 1,000 personas) los flujos proceden de Choluteca hacia Tegucigalpa.

En resumen, la región sur los niveles de arraigo hasta el año 2001 demuestran que la población nativa de la misma solo el $72 \%$ vive en ella, teniendo un porcentaje alto que vive en otros municipios del país.

E\&A

\subsubsection{Migración internacional.}

La región sur de Honduras en los últimos años no ha sido una región atractiva desde el punto de vista de la inmigración internacional, En el último censo de población del 2001 la región recibe limitados flujos de inmigrantes, que en su mayoría son de algunos países centroamericanos, principalmente Nicaragua y El Salvador. Otro país que aporta es Estados Unidos de América (Tabla No.4).

\section{Tabla No.4}

Red Sur UNAH: Población según País de Nacimiento, 2001

\begin{tabular}{|l|r|r|}
\hline \multicolumn{1}{|c|}{ País de Nacimiento } & \multicolumn{1}{c|}{ Total } & \multicolumn{1}{c|}{$\%$} \\
\hline Nicaragua & 754 & 46.8 \\
\hline El Salvador & 501 & 31.1 \\
\hline Guatemala & 66 & 4.1 \\
\hline Costa Rica & 10 & 0.6 \\
\hline Panamá & 6 & 0.4 \\
\hline Belice & 1 & 0.1 \\
\hline Estados Unidos de América & 129 & 8.0 \\
\hline México & 21 & 1.3 \\
\hline
\end{tabular}




\begin{tabular}{|l|r|r|}
\hline \multicolumn{1}{|c|}{ País de Nacimiento } & \multicolumn{1}{c|}{ Total } & \multicolumn{1}{c|}{$\%$} \\
\hline Canadá & 14 & 0.9 \\
\hline Sur América & 46 & 0.9 \\
\hline Caribe & 17 & 1.1 \\
\hline Europa & 14 & 0.9 \\
\hline Asia & 19 & 1.2 \\
\hline Ignorado & 12 & 0.7 \\
\hline Total & 1610 & 100.0 \\
\hline
\end{tabular}

(Fuente: Elaboración propia en base a datos censales del CNPV 2001, INE, Honduras)

Tabla No.5

Red Sur UNAH: Hogares con Emigrantes Internacionales según municipio, 2001

\begin{tabular}{|l|r|r|r|r|r|r|}
\hline \multicolumn{1}{|c|}{ Municipio } & \multicolumn{1}{c|}{$\mathrm{Si}$} & \multicolumn{1}{c|}{ No } & \multicolumn{1}{c|}{ Total } & \multicolumn{1}{l}{ Si } & \multicolumn{1}{l}{ No } & \multicolumn{1}{c|}{ Total } \\
\hline Choluteca & 789 & 23,156 & 23,945 & 3.30 & 96.70 & 100.00 \\
\hline Apacilagua & 14 & 1,666 & 1,680 & 0.83 & 99.17 & 100.00 \\
\hline Concepción de Maria & 33 & 4,350 & 4,383 & 0.75 & 99.25 & 100.00 \\
\hline Duyure & 1 & 608 & 609 & 0.16 & 99.84 & 100.00 \\
\hline El Corpus & 52 & 3,998 & 4,050 & 1.28 & 98.72 & 100.00 \\
\hline El Triunfo & 101 & 6,391 & 6,492 & 1.56 & 98.44 & 100.00 \\
\hline Marcovia & 615 & 6,839 & 7,454 & 8.25 & 91.75 & 100.00 \\
\hline Morolica & 16 & 919 & 935 & 1.71 & 98.29 & 100.00 \\
\hline Namasigue & 46 & 4,474 & 4,520 & 1.02 & 98.98 & 100.00 \\
\hline Orocuina & 34 & 3,106 & 3,140 & 1.08 & 98.92 & 100.00 \\
\hline Pespire & 109 & 4,363 & 4,472 & 2.44 & 97.56 & 100.00 \\
\hline San Antonio de Flores & 6 & 1,039 & 1,045 & 0.57 & 99.43 & 100.00 \\
\hline San Isidro & 14 & 685 & 699 & 2.00 & 98.00 & 100.00 \\
\hline San José & 7 & 637 & 644 & 1.09 & 98.91 & 100.00 \\
\hline San Marcos de Colón & 55 & 3,911 & 3,966 & 1.39 & 98.61 & 100.00 \\
\hline Santa Ana de Yusguare & 17 & 1,935 & 1,952 & 0.87 & 99.13 & 100.00 \\
\hline Total Choluteca & 1,909 & 68,077 & 69,986 & 2.73 & 97.27 & 100.00 \\
\hline Nacaome & 653 & 8,556 & 9,209 & 7.09 & 92.91 & 100.00 \\
\hline Alianza & 234 & 1,370 & 1,604 & 14.59 & 85.41 & 100.00 \\
\hline Amapala & 94 & 1,867 & 1,961 & 4.79 & 95.21 & 100.00 \\
\hline Aramecina & 91 & 986 & 1,147 & 14.04 & 85.96 & 100.00 \\
\hline
\end{tabular}

E\&A 


\begin{tabular}{|l|r|r|r|r|r|r|}
\hline \multicolumn{1}{|c|}{ Municipio } & \multicolumn{1}{c|}{$\mathrm{Si}$} & \multicolumn{1}{c|}{ No } & \multicolumn{1}{c|}{ Total } & \multicolumn{1}{c|}{ Si } & \multicolumn{1}{l|}{ No } & \multicolumn{1}{c|}{ Total } \\
\hline Caridad & 9 & 662 & 671 & 1.34 & 98.66 & 100.00 \\
\hline Goascorán & 185 & 2,439 & 2,624 & 7.05 & 92.95 & 100.00 \\
\hline Langue & 78 & 3,287 & 3,365 & 2.32 & 97.68 & 100.00 \\
\hline San Francisco de Coray & 75 & 1,462 & 1,537 & 4.88 & 95.12 & 100.00 \\
\hline San Lorenzo & 255 & 5,379 & 5,634 & 4.53 & 95.47 & 100.00 \\
\hline Total Valle & 1,744 & 26,008 & 27,752 & 6.28 & 93.72 & 100.00 \\
\hline Total red Sur UNAH & 3,653 & 94,085 & 97,738 & 3.74 & 96.26 & 100.00 \\
\hline
\end{tabular}

(Fuente: Elaboración propia en base a datos censales del CNPV 2001, INE, Honduras)

E\&A

En cambio, la región sur se convirtió en la zona de expulsión, no solo a nivel interno, sino a nivel internacional. Datos censales del 2001 corroboran esta afirmación y muestran que en el departamento de Valle existe una mayor predisposición a emigrar al exterior, principalmente en aquellos municipios limítrofes o cercanos con la república de El Salvador (Alianza, Aramecina, Langue, Nacaome), donde hay una mayor experiencia emigratoria hacia el exterior, principalmente hacia los Estados Unidos de América (Tabla No.5).

\subsection{Distribución espacial de la población.}

El cuadro No.6 nos muestra que en las últimas dos décadas se han dado cambios en la urbanización de la región sur. La población urbana ha aumentado un diez por ciento, de 24\% en 1988 ha aumentado a un $34 \%$ en el 2011, aunque ocupa un cuarto lugar al compararla con las nueve redes educativas de la UNAH que está dividido el país y por debajo del promedio nacional. 
Tabla No.6

Red Sur UNAH: Población Total por Área de Residencia según Municipio, 1988-2011

\begin{tabular}{|c|c|c|c|c|c|c|}
\hline Municipio & $\begin{array}{c}\text { Urbano } \\
1988\end{array}$ & $\begin{array}{c}\text { Rural } \\
1988\end{array}$ & $\begin{array}{c}\text { Urbano } \\
2001\end{array}$ & $\begin{array}{l}\text { Rural } \\
2001\end{array}$ & $\begin{array}{c}\text { Urbano } \\
2011\end{array}$ & $\begin{array}{l}\text { Rural } \\
2011\end{array}$ \\
\hline Choluteca & 61.08 & 38.92 & 62.49 & 37.51 & 66.71 & 33.29 \\
\hline Apacilagua & 0.00 & 100.00 & 0.00 & 100.00 & 0.00 & 100.00 \\
\hline Concepción de María & 0.00 & 100.00 & 0.00 & 100.00 & 0.00 & 100.00 \\
\hline Duyure & 0.00 & 100.00 & 0.00 & 100.00 & 0.00 & 100.00 \\
\hline El Corpús & 0.00 & 100.00 & 0.00 & 100.00 & 0.00 & 100.00 \\
\hline El Triunfo & 17.25 & 82.75 & 18.36 & 81.64 & 21.48 & 78.52 \\
\hline Marcovia & 17.00 & 83.00 & 17.08 & 82.92 & 19.20 & 80.80 \\
\hline Morolica & 0.00 & 100.00 & 0.00 & 100.00 & 0.00 & 100.00 \\
\hline Namasigue & 0.00 & 100.00 & 9.03 & 90.97 & 10.74 & 89.26 \\
\hline Orocuina & 0.00 & 100.00 & 0.00 & 100.00 & 0.00 & 100.00 \\
\hline Pespire & 9.74 & 90.26 & 12.48 & 87.52 & 16.83 & 83.17 \\
\hline San Antonio de Flores & 0.00 & 100.00 & 0.00 & 100.00 & 0.00 & 100.00 \\
\hline San Isidro & 0.00 & 100.00 & 0.00 & 100.00 & 0.00 & 100.00 \\
\hline San José & 0.00 & 100.00 & 0.00 & 100.00 & 0.00 & 100.00 \\
\hline San Marcos de Colón & 37.73 & 62.27 & 41.06 & 58.94 & 47.03 & 52.97 \\
\hline Santa Ana de Yusguare & 0.00 & 100.00 & 0.00 & 100.00 & 0.00 & 100.00 \\
\hline Total Choluteca & 24.59 & 75.41 & 28.00 & 72.00 & 33.25 & 66.75 \\
\hline Nacaome & 25.22 & 74.78 & 34.32 & 65.68 & 44.47 & 55.53 \\
\hline Alianza & 0.00 & 100.00 & 0.00 & 100.00 & 0.00 & 100.00 \\
\hline Amapala & 30.46 & 69.54 & 22.74 & 77.26 & 19.47 & 80.53 \\
\hline Aramecina & 0.00 & 100.00 & 0.00 & 100.00 & 0.00 & 100.00 \\
\hline Caridad & 0.00 & 100.00 & 0.00 & 100.00 & 0.00 & 100.00 \\
\hline Goascorán & 0.00 & 100.00 & 0.00 & 100.00 & 0.00 & 100.00 \\
\hline Langue & 18.42 & 81.58 & 19.03 & 80.97 & 21.24 & 78.76 \\
\hline San Francisco de Coray & 0.00 & 100.00 & 0.00 & 100.00 & 0.00 & 100.00 \\
\hline San Lorenzo & 73.50 & 26.50 & 72.25 & 27.75 & 73.45 & 26.55 \\
\hline Total Valle & 25.42 & 74.58 & 29.91 & 70.09 & 36.06 & 63.94 \\
\hline Total Región Sur UNAH & 24.83 & 75.17 & 28.54 & 71.46 & 34.01 & 65.99 \\
\hline
\end{tabular}

(Fuente: Elaboración propia en base a datos censales y Proyecciones de Población de Honduras, 
San Lorenzo y Choluteca son municipios que pueden considerarse altamente urbanizados, $73 \%$ y $67 \%$ respectivamente. De allí hay dos municipios con aspiraciones a superar la mitad de su población en áreas urbanas, otros siete municipios donde la urbanización es incipiente y por último se encuentran un total de quince municipios donde la urbanización es cero según la definición de comunidades urbanas vigentes en el país.

\subsection{Proyecciones de la población.}

La población de la región sur continuará creciendo en el futuro inmediato. Las proyecciones muestran como en los próximos cinco años alcanzará un total de 682,890 personas (INE, 2006) con una mortalidad y fecundidad en descenso lento y un patrón migratorio que se dirige principalmente hacia Tegucigalpa y a los Estados Unidos de América (Tabla No.7).

Tabla No.7

E\&A IIES

Red Sur UNAH: Proyecciones de Población según Municipio, 2001-2015

\begin{tabular}{|c|c|c|c|c|c|}
\hline Municipio & 2011 & 2012 & 2013 & 2014 & 2015 \\
\hline Choluteca & 174,039 & 178,779 & 183,563 & 188,383 & 193,230 \\
\hline Apacilagua & 9,532 & 9,523 & 9,513 & 9,504 & 9,494 \\
\hline Concepción de María & 26,913 & 26,981 & 27,049 & 27,118 & 27,185 \\
\hline Duyure & 2,848 & 2,837 & 2,826 & 2,815 & 2,804 \\
\hline El Corpús & 23,490 & 23,488 & 23,487 & 23,485 & 23,482 \\
\hline El Triunfo & 47,715 & 48,723 & 49,746 & 50,782 & 51,832 \\
\hline Marcovia & 48,935 & 49,820 & 50,715 & 51,618 & 52,528 \\
\hline Morolica & 5,763 & 5,799 & 5,836 & 5,873 & 5,911 \\
\hline Namasigue & 32,146 & 32,702 & 33,266 & 33,837 & 34,413 \\
\hline Orocuina & 17,601 & 17,648 & 17,694 & 17,740 & 17,786 \\
\hline Pespirre & 25,762 & 25,833 & 25,904 & 25,975 & 26,044 \\
\hline San Antonio de Flores & 6,049 & 6,080 & 6,111 & 6,142 & 6,173 \\
\hline San Isidro & 3,564 & 3,556 & 3,549 & 3,541 & 3,534 \\
\hline San José & 3,802 & 3,818 & 3,833 & 3,849 & 3,865 \\
\hline San Marcos de Colón & 24,983 & 25,267 & 25,548 & 25,824 & 26,095 \\
\hline Santa Ana de Yusguare & 13,947 & 14,290 & 14,642 & 15,002 & 15,371 \\
\hline Total Choluteca & 467,088 & 475,144 & 483,282 & 491,489 & 499,747 \\
\hline Nacaome & 59,512 & 60,569 & 61,637 & 62,712 & 63,791 \\
\hline
\end{tabular}




\begin{tabular}{|r|r|r|r|r|r|}
\hline \multicolumn{1}{|c|}{ Municipio } & \multicolumn{1}{|c|}{2011} & \multicolumn{1}{c|}{2012} & \multicolumn{1}{c|}{2013} & \multicolumn{1}{c|}{2014} & \multicolumn{1}{|c|}{2015} \\
\hline Alianza & 6,473 & 6,379 & 6,284 & 6,187 & 6,089 \\
\hline Amapala & 12,023 & 12,190 & 12,355 & 12,517 & 12,675 \\
\hline Aramecina & 6,469 & 6,464 & 6,457 & 6,446 & 6,433 \\
\hline Caridad & 3,735 & 3,726 & 3,716 & 3,704 & 3,691 \\
\hline Goascorán & 14,036 & 14,007 & 13,972 & 13,932 & 13,885 \\
\hline Langue & 21,802 & 21,994 & 22,178 & 22,353 & 22,518 \\
\hline San Fco. De Coray & 10,032 & 10,116 & 10,197 & 10,275 & 10,347 \\
\hline San Lorenzo & 39,858 & 40,824 & 41,791 & 42,756 & 43,715 \\
\hline Total Valle & 173,941 & 176,269 & 178,587 & 180,882 & 183,143 \\
\hline Total Región Sur UNAH & 641,029 & 651,413 & 661,869 & 672,371 & 682,890 \\
\hline
\end{tabular}

(Fuente: Proyecciones de Población de Honduras, INE.)

\subsection{Población en edad de educación terciaria.}

La población total de la región ha aumentado en un 38\% tomando como base desde 1988 hasta el 2011; en cambio la población en edad de educación terciaria aumentó un 54\% en el mismo periodo.

El peso de la población terciaria con respecto al total de la población aumentó un 3.7 por ciento, pasando de 10.7\% en 1988 a 14.4\% en el 2011 (Flores, 2011). En cuanto al índice de masculinidad en la población objetivo hay una tendencia al cambio de mayoría de hombres por mujeres a través del tiempo (Tabla No.8).

Tabla No.8

Red Sur UNAH: Población en Educación Terciaria, 1988-2015

\begin{tabular}{|l|r|r|r|r|}
\hline \multicolumn{1}{|c|}{ Indicador } & \multicolumn{1}{c|}{1988} & \multicolumn{1}{c|}{2001} & \multicolumn{1}{c|}{2011} & \multicolumn{1}{c|}{2015} \\
\hline Población Total de la región & 399,034 & 506,495 & 641,029 & 682,890 \\
\hline Indice de Masculinidad Total & 99.1 & 98.7 & 98.3 & 98.4 \\
\hline Población de 18-24 años & 42,583 & 64,327 & 92,319 & 98,303 \\
\hline \% poblacion de 18-24 años del total & 10.7 & 12.7 & 14.4 & 14.4 \\
\hline Indice de Masculinidad 18-24 años & $93 . .78$ & 95.6 & 103.8 & 102.4 \\
\hline
\end{tabular}

(Fuente: Elaboración propia en base a datos censales y proyecciones, DGECH e INE.)

De la población en edad de educación terciaria en la región sur los mayores volúmenes se encuentran 
$\mathbf{E \& A}$ IIES

en los municipios más poblados, entre ellos Choluteca y en mucha menor escala Nacaome, Marcovia, San Lorenzo y El Triunfo. Hay municipios donde la población en edad de educación superior es muy limitada e incluso con estos datos no se sabe si ha superado o no la educación media como insumo requerido de los estudios universitarios (Tabla No.9).

Tabla No.9

Región Sur UNAH: Población Total y en Edad de Educación Terciaria según Municipio 2011-2015

\begin{tabular}{|c|c|c|c|c|c|c|c|c|}
\hline \multirow[b]{2}{*}{ Municipios } & \multicolumn{2}{|c|}{2011} & \multicolumn{2}{|c|}{2015} & \multicolumn{2}{|c|}{2011} & \multicolumn{2}{|c|}{2015} \\
\hline & $\begin{array}{c}\text { Población } \\
\text { Total }\end{array}$ & $\begin{array}{c}18-24 \\
\text { años }\end{array}$ & $\begin{array}{l}\text { Población } \\
\text { total }\end{array}$ & $\begin{array}{c}18-24 \\
\text { años }\end{array}$ & $\begin{array}{c}\text { Población } \\
\text { Total }\end{array}$ & $\begin{array}{c}18-24 \\
\text { años }\end{array}$ & $\begin{array}{l}\text { Población } \\
\text { total }\end{array}$ & $\begin{array}{c}18-24 \\
\text { años }\end{array}$ \\
\hline Choluteca & 174,039 & 26,775 & 193,230 & 29,950 & 27.15 & 29.00 & 28.30 & 30.47 \\
\hline Apacilagua & 9,532 & 1,204 & 9,494 & 1,219 & 1.49 & 1.30 & 1.39 & 1.24 \\
\hline Concepción de María & 26,913 & 3,498 & 27,185 & 3,595 & 4.20 & 3.79 & 3.98 & 3.66 \\
\hline Duyure & 2,848 & 319 & 2,804 & 319 & 0.44 & 0.35 & 0.41 & 0.32 \\
\hline El Corpus & 23,490 & 3,103 & 23,482 & 3,150 & 3.66 & 3.36 & 3.44 & 3.20 \\
\hline El Triunfo & 47,715 & 6,177 & 51,832 & 6,822 & 7.44 & 6.69 & 7.59 & 6.94 \\
\hline Marcovia & 48,935 & 7,421 & 52,528 & 8,094 & 7.63 & 8.04 & 7.69 & 8.23 \\
\hline Morolica & 5,763 & 754 & 5,911 & 783 & 0.90 & 0.82 & 0.87 & 0.80 \\
\hline Namasigue & 32,146 & 4,607 & 34,413 & 5,021 & 5.01 & 4.99 & 5.04 & 5.11 \\
\hline Orocuina & 17,601 & 2,272 & 17,786 & 2,332 & 2.75 & 2.46 & 2.60 & 2.37 \\
\hline Pespire & 25,762 & 3,061 & 26,044 & 3,142 & 4.02 & 3.32 & 3.81 & 3.20 \\
\hline San Antonio de Flores & 6,049 & 772 & 6,173 & 800 & 0.94 & 0.84 & 0.90 & 0.81 \\
\hline San Isidro & 3,564 & 395 & 3,534 & 397 & 0.56 & 0.43 & 0.52 & 0.40 \\
\hline San José & 3,802 & 366 & 3,865 & 379 & 0.59 & 0.40 & 0.57 & 0.39 \\
\hline San Marcos de Colón & 24,983 & 3,332 & 26,095 & 3,509 & 3.90 & 3.61 & 3.82 & 3.57 \\
\hline Santa Ana de Yusguare & 13,947 & 1,942 & 15,371 & 2,173 & 2.18 & 2.10 & 2.25 & 2.21 \\
\hline Nacaome & 59,512 & 9,100 & 63,791 & 9,410 & 9.28 & 9.86 & 9.34 & 9.57 \\
\hline Alianza & 6,473 & 896 & 6,089 & 788 & 1.01 & 0.97 & 0.89 & 0.80 \\
\hline Amapala & 12,023 & 1,811 & 12,675 & 1,802 & 1.88 & 1.96 & 1.86 & 1.83 \\
\hline Aramecina & 6,469 & 878 & 6,433 & 820 & 1.01 & 0.95 & 0.94 & 0.83 \\
\hline Caridad & 3,735 & 543 & 3,691 & 503 & 0.58 & 0.59 & 0.54 & 0.51 \\
\hline Goascorán & 14,036 & 1,966 & 13,885 & 1,821 & 2.19 & 2.13 & 2.03 & 1.85 \\
\hline Langue & 21,802 & 2,900 & 22,518 & 2,842 & 3.40 & 3.14 & 3.30 & 2.89 \\
\hline San Francisco de Coray & 10,032 & 1,346 & 10,347 & 1,307 & 1.56 & 1.46 & 1.52 & 1.33 \\
\hline San Lorenzo & 39,858 & 6,880 & 43,715 & 7,323 & 6.22 & 7.45 & 6.40 & 7.45 \\
\hline Total & 641,029 & 92,319 & 682,890 & 98,303 & 100.00 & 100.00 & 100.00 & 100.00 \\
\hline
\end{tabular}

(Fuente: Elaboración propia en base a datos de Proyecciones de Población, INE.) 
En cuanto al área de residencia la población en edad objetivo de la educación terciaria muestra como la tendencia de los flujos de este grupo de edad es mayoritariamente rural, e incluso llegan a ser el doble de los urbanos. Sin embargo, la educación superior es eminentemente urbana y es en esa área de residencia donde hay mayor acceso a los niveles previo a la educación superior que son los niveles secundarios (Tabla No.10).

Dada esa realidad las poblaciones de Choluteca, San Lorenzo y Nacaome son los que podrían tener mayores posibilidades de acceso a la educación superior por ser un mayor mercado potencial, sin embargo, solo en el primero la oferta educativa superior está más consolidada, aunque la cercanía geográfica, las facilidades de transporte, pueden incidir para que la cobertura sea más amplia partiendo de un nodo principal a través de una estrategia de educación superior más activa, enfocada no solo en el área urbana sino en las áreas rurales de la red.

Tabla No.10

\section{Red Sur UNAH: Población en Edad de Educación Terciaria por Área de Residencia según}

Municipio, 1988- 2011.

\begin{tabular}{|l|r|r|r|r|r|r|r|r|r|}
\hline Municipio & $\begin{array}{c}\text { Urbano } \\
1988\end{array}$ & $\begin{array}{c}\text { Rural } \\
1988\end{array}$ & $\begin{array}{c}\text { Total } \\
1988\end{array}$ & $\begin{array}{c}\text { Urbano } \\
2001\end{array}$ & $\begin{array}{c}\text { Rural } \\
2001\end{array}$ & $\begin{array}{c}\text { Total } \\
2001\end{array}$ & $\begin{array}{c}\text { Urbano } \\
2011\end{array}$ & $\begin{array}{c}\text { Rural } \\
2011\end{array}$ & $\begin{array}{c}\text { Total } \\
2011\end{array}$ \\
\hline Choluteca & 6,689 & 3,281 & 9,970 & 11,435 & 6,044 & 17,479 & 18,103 & 8,671 & 26,774 \\
\hline Apacilagua & & 856 & 856 & 0 & 1,005 & 1,005 & & 1,204 & 1,204 \\
\hline $\begin{array}{l}\text { Concepción } \\
\text { de María }\end{array}$ & & 2,201 & 2,201 & 0 & 2,819 & 2,819 & & 3,498 & 3,498 \\
\hline Duyure & & 288 & 288 & 0 & 275 & 275 & & 319 & 319 \\
\hline El Corpus & & 2,113 & 2,113 & 0 & 2,578 & 2,578 & & 3,103 & 3,103 \\
\hline El Triunfo & 475 & 2,250 & 2,725 & 832 & 3,341 & 4,173 & 1,352 & 4,826 & 6,178 \\
\hline Marcovia & 595 & 2,618 & 3,213 & 928 & 4,251 & 5,179 & 1,408 & 6,013 & 7,421 \\
\hline Morolica & & 403 & 403 & 0 & 585 & 585 & & 754 & 754 \\
\hline Namasigue & & 1,802 & 1,802 & 322 & 2,899 & 3,221 & 513 & 4,094 & 4,607 \\
\hline Orocuina & & 1,358 & 1,358 & 0 & 1,838 & 1,838 & & 2,272 & 2,272 \\
\hline Pespire & 240 & 1,748 & 1,988 & 355 & 2,128 & 2,483 & 547 & 2,514 & 3,061 \\
\hline
\end{tabular}

E\&A IIES 


\begin{tabular}{|c|c|c|c|c|c|c|c|c|c|}
\hline Municipio & $\begin{array}{c}\text { Urbano } \\
1988\end{array}$ & $\begin{array}{c}\text { Rural } \\
1988\end{array}$ & $\begin{array}{l}\text { Total } \\
1988\end{array}$ & $\begin{array}{c}\text { Urbano } \\
2001\end{array}$ & $\begin{array}{c}\text { Rural } \\
2001\end{array}$ & $\begin{array}{l}\text { Total } \\
2001\end{array}$ & $\begin{array}{c}\text { Urbano } \\
2011\end{array}$ & $\begin{array}{c}\text { Rural } \\
2011\end{array}$ & $\begin{array}{l}\text { Total } \\
2011\end{array}$ \\
\hline $\begin{array}{l}\text { San } \\
\text { Antonio de } \\
\text { Flores }\end{array}$ & & 412 & 412 & 0 & 608 & 608 & & 772 & 772 \\
\hline San Isidro & & 303 & 303 & 0 & 333 & 333 & & 395 & 395 \\
\hline San José & & 227 & 227 & 0 & 288 & 288 & & 366 & 366 \\
\hline $\begin{array}{l}\text { San Marcos } \\
\text { de Colón }\end{array}$ & 755 & 1,110 & 1,865 & 1,090 & 1,442 & 2,532 & 1,563 & 1,769 & 3,332 \\
\hline $\begin{array}{l}\text { Santa Ana } \\
\text { de Yusguare }\end{array}$ & & 713 & 713 & 0 & 1,270 & 1,270 & & 1,942 & 1,942 \\
\hline $\begin{array}{l}\text { Total } \\
\text { Choluteca }\end{array}$ & 8,754 & 21,683 & 30,437 & 14,962 & 31,704 & 46,666 & 23,486 & 42,512 & 65,998 \\
\hline Nacaome & 1,095 & 2,798 & 3,893 & 2,152 & 3,721 & 5,873 & 4,299 & 4,801 & 9,100 \\
\hline Alianza & & 755 & 755 & 0 & 807 & 807 & & 896 & 896 \\
\hline Amapala & 271 & 601 & 872 & 290 & 933 & 1,223 & 369 & 1,442 & 1,811 \\
\hline Aramecina & & 549 & 549 & 0 & 687 & 687 & & 878 & 878 \\
\hline Caridad & & 323 & 323 & 0 & 436 & 436 & & 543 & 543 \\
\hline Goascorán & & 1,218 & 1,218 & 0 & 1,562 & 1,562 & & 1,966 & 1,966 \\
\hline Langue & 240 & 1,334 & 1,574 & 400 & 1,628 & 2,028 & 639 & 2,261 & 2,900 \\
\hline $\begin{array}{l}\text { San } \\
\text { Francisco } \\
\text { de Coray }\end{array}$ & & 648 & 648 & 0 & 949 & 949 & & 1,346 & 1,346 \\
\hline San Lorenzo & 1,793 & 521 & 2,314 & 2,935 & 1,161 & 4,096 & 5,023 & 1,856 & 6,879 \\
\hline Total Valle & 3,399 & 8,747 & 12,146 & 5,777 & 11,884 & 17,661 & 10,330 & 15,989 & 26,319 \\
\hline $\begin{array}{l}\text { Total Red } \\
\text { Sur UNAH }\end{array}$ & 12,153 & 30,430 & 42,583 & 20,739 & 43,588 & 64,327 & 33,816 & 58,501 & 92,317 \\
\hline
\end{tabular}

(Fuente: Elaboración propia en base de datos censales y Proyecciones de Población, DGEC e INE.) 


\section{CONCLUSIONES}

- La población de la red sur de la UNAH, que incluye los departamentos de Choluteca y Valle, ha experimentado un crecimiento acelerado desde los años cincuenta. El volumen de la población aumentó de ciento ochenta mil hasta alcanzar en el 2011una población que supera las seiscientas mil personas, es decir aumentó casi cuatro veces.

- La estructura de la población es eminentemente joven, donde más de un tercio es menor de quince años, pero el mayor porcentaje está ubicado en la población activa ( 15 a 64 años) que supera la mitad de la población (55\%). Actualmente la RDT indica que hay 78 personas dependientes por cada cien personas en edad productiva.

- Los niveles de fecundidad y mortalidad son altos si los comparamos con los de otras regiones del país con mayor desarrollo relativo y más con otras realidades internacionales. La esperanza de vida al nacer ha tenido mejoras en el tiempo, principalmente en la última década.

- En la región sur la población en las últimas tres décadas ha mostrado tener saldos netos de migración interna interdepartamental negativos, es decir, es mayormente una región de expulsión de población, y a través del tiempo este patrón se ha incrementado en flujo. De la región sur, en la migración absoluta hasta el año 2001 habían salido 152,680 personas y habían llegado solo 26,469 personas para tener un saldo migratorio negativo interdepartamental de 126,211 personas.

- En las últimas décadas la región sur de Honduras no ha sido una región atractiva desde el punto de vista de la inmigración internacional. Se convirtió en zona de expulsión, para el caso el departamento de Valle tiene una mayor predisposición en la región sur a emigrar al exterior, 'principalmente en aquellos municipios limítrofes o cercanos con la república de El Salvador (Alianza, Aramecina, Langue, Nacaome), donde hay una mayor experiencia emigratoria hacia el exterior, principalmente hacia los Estados Unidos de América.

- En un futuro inmediato, la población de la región sur continuará creciendo, proyectándose que para el 2015 un total de 682,890 personas, en la conjugación de una mortalidad y fecundidad en descenso lento y un patrón migratorio que se dirige internamente hacia Tegucigalpa e internacionalmente hacia los Estados Unidos de América.

- Los mayores volúmenes de la población en edad de educación terciaria en la región sur a nivel municipal se encuentra en los municipios más poblados, entre ellos sobresale Choluteca y en 
mucha menor medida Nacaome, Marcovia, San Lorenzo y El Triunfo.

- Las poblaciones en edad objetivo de la educación terciaria por área de residencia son mayoritariamente rurales. Sin embargo, como la oferta de la educación superior es eminentemente urbana, las poblaciones de los municipios de Choluteca, San Lorenzo, Nacaome son los que tienen mayores posibilidades de acceso por tener un mayor mercado potencial inmediato, en particular Choluteca donde la oferta educativa está más consolidada. 


\section{REFERENCIAS}

Flores, Manuel. 2004. Estado de la Población, Tegucigalpa, Instituto de Investigaciones Económicas y Sociales (IIES), Universidad Nacional Autónoma de Honduras (UNAH), 2004.

Flores, Manuel, 2005. Migración interna intermunicipal de Honduras, Tegucigalpa, Instituto de Investigaciones Económicas y Sociales (IIES), Universidad Nacional Autónoma de Honduras (UNAH).

Flores, Manuel. 2011. Marco Contextual Demográfico de la Educación Superior en Honduras, Tegucigalpa, Instituto de Investigaciones Económicas y Sociales (IIES), Universidad Nacional Autónoma de Honduras (UNAH), 2011.

INE, 2006. Honduras: Proyecciones de Población 2001-2050, Tomo 1, Proyecciones de población total, urbana y rural por sexo y edad 2001-2015 y total quinquenales 2015-2050, Tegucigalpa, INE.

UNAH, 2008. Redes Educativas Regionales de la UNAH para la Gestión del Conocimiento con Calidad, Pertinencia y Equidad, Serie de Publicaciones de la Reforma Universitaria No.2, Tegucigalpa, UNAH.

UNAH, 2010. Propuesta de Proyecto "Oferta y Demanda de la Educación Superior de Honduras: Sistema Integrado de Valor y de Suministro (SIVSES), Tegucigalpa, Instituto de Investigaciones Económicas y Sociales (IIES), Universidad Nacional Autónoma de Honduras (UNAH). 
Vol. 3
$N^{\circ} 1$
2012

E\&A

IIES 